\title{
ON UNAVERAGED CONVERGENCE OF POSITIVE OPERATORS IN LEBESGUE SPACE
}

\author{
BY \\ H. FONG(1) AND L. SUCHESTON(2)
}

\begin{abstract}
Let $T$ be a power-bounded positive conservative operator on $L_{1}$ of a $\sigma$ finite measure space. Let $e$ be a bounded positive function invariant under the operator adjoint to $T$.

Theorem. (1) $\int\left|T^{n} f\right| \cdot e \rightarrow 0$ implies (2) $\int\left|T^{n} f\right| \rightarrow 0$.

If $T$ and all its powers are ergodic, and $T$ satisfies an abstract Harris condition, then (1) holds by the Jamison-Orey theorem for all integrable $f$ with $\int f \cdot e=0$, and hence also (2) holds for $\operatorname{such} f$. A new proof of the Jamison-Orey theorem is given, via the 'filling scheme'. For discrete measure spaces this is due to Donald Ornstein, Proc. Amer. Math. Soc. 22 (1969), 549-551. If $T$ is power-bounded, conservative and ergodic, and $0<f_{0}=T f_{0}$, then $f_{0}: e \in L_{1}$ implies $f_{0} \in L_{1}$, hence (2) implies that $T^{n} f$ converges for each $f \in L_{1}$.

Theorem. Let $T$ be a positive conservative contraction on $L_{1}$; then the class of functions $\left\{f-T f, f \in L_{1}^{+}\right\}$is dense in the class of functions $\left\{f-T f, f \in L_{1}\right\}$.
\end{abstract}

1. Let $(X, \mathcal{A}, m)$ be a $\sigma$-finite measure space. We consider a positive linear operator $T$ on $L_{1}$, such that $\sup _{n}\left|T^{n}\right|_{1}<\infty$. It is known [13] that $X$ decomposes into sets $Y$ and $Z$, such that $f \in L_{1}^{+}(Y)$ implies $\varliminf_{n} \int T^{n} f>0 ; f \in L_{1}(Z)$ implies $T f \in L_{1}(Z)$ and $\lim _{n} \int\left|T^{n} f\right|=0$; moreover, there exists a function $e \in L_{\infty}^{+}$such that supp $e=Y$ and $T^{*} e=e$. If $T$ is conservative and ergodic, i.e., $f \geq 0, f \not 0$ implies $\sum_{i=0}^{\infty} T^{i} f=\infty$ a.e., then either $X=Y$ or $X=Z$. We investigate in the present paper the convergence to zero of $\left|T^{n} f\right|_{1}$. If $X=Z$, there is nothing to investigate since the convergence to zero is assured for all $f$; therefore we may and do assume $X=Y$. The papers of Orey [8] and Jamison and Orey [5] discuss the same problem for $T$ Markovian and in a probabilistic context. Assuming that the measure is discrete, D. S. Ornstein [9] showed how to reduce the present situation to the Markovian case. Here this is done for general measure spaces and without assuming $T$ ergodic (Theorem 2.1). We give in all detail the deep arguments only sketched by Ornstein. Since we do not assume that the measure is discrete or $T$ is ergodic, our proof is more involved, and we need also to appeal to the theorems of H. Rost [12] giving conditions for the

Presented to the Society, January 17, 1972; received by the editors October 18, 1971 and, in revised form, May 12, 1972.

AMS (MOS) subject classifications (1970). Primary 60F15, 28A65; Secondary 60J05.

Key words and phrases. Positive operator, conservative and ergodic, invariant function, $L_{1}$ convergence, transition measure, Harris condition.

(1) The research of this author was supported by a grant from the Bowling Green State University.

(2) The research of this author was in part supported by the National Science Foundation under grant GP 13692. 
'filling scheme' to terminate in the limit. A variant of this argument is also used to give a new and nonprobabilistic proof of the Jamison-Orey theorem (Theorem 4.1); Ornstein [9] sketched such a proof of Orey's theorem (discrete case of Jamison-Orey). The argument also yields a strengthening of an $L_{1}$ decomposition theorem due to Chacon [1]. Our result (Theorem 2.4) may be briefly stated as follows: If $T$ is a positive conservative contraction then the classes of functions $\left\{f-T f: f \in L_{1}\right\}$ and $\left\{f-T f: f \in L_{1}^{+}\right\}$have identical closures in $L_{1}$. Y. Derriennic and $M$. Lin having read a first version of this paper produced a shorter proof of Theorem 2.1, based in the ergodic case on the observation that the $T^{*}$-invariant function $e$ may be identified with $\lim _{n} \sup T^{* n} 1$. The argument given here nevertheless has the advantage of allowing unified proofs of Theorems 2.1, 2.4, and 4.1.

As an application of Theorem 2.1 we obtain a 'power-bounded' non-Markovian version of the Jamison-Orey theorem (Theorem 3.1). For a probabilistic interpretation of positive non-Markovian operators, see e.g. the book The Theory of Branching Processes by T. E. Harris. Because of the existence of such an interpretation, we believe it worthwhile to also give limit theorems for powerbounded non-Markovian transition measures (Theorems 3.2 and 3.3).

2. Let $(X, \AA, m)$ be a $\sigma$-finite measure space. $L_{1}(m)$ denotes the space of realvalued integrable functions on $(X, \mathcal{A}, m)$. All sets and functions below are assumed measurable; all relations are assumed to hold modulo sets of $m$-measure zero. Let $T$ be a positive linear operator on $L_{1}(m)$. Assume that $T$ is powerbounded:

$$
\sup _{n}\left|T^{n}\right|_{1}=M<\infty
$$

and conservative:

$$
f \in L_{1}^{+}, f \not 0 \text { implies } \sum_{i=0}^{\infty} T^{i} f=\infty \text { or } 0 \text { on } X .
$$

We also assume that $X=Y$; i.e.,

$$
\text { there exists a function } e \in L_{\infty}^{+} \text {such that } T^{*} e=e>0 \text { on } X \text {. }
$$

As remarked in $\S 1$, the assumption (2.3) can be made without loss of generality if $T$ is conservative and ergodic. However, the following theorem does not assume ergodicity.

Theorem 2.1. Let $T$ satisfy (2.1), (2.2) and (2.3). If $f \in L_{1}(m)$ and $\lim _{n} \int\left|T^{n} f\right| e d m=0$ then $\lim _{n} \int\left|T^{n} f\right| d m=0$.

Proof. We introduce a new measure $v$ defined by $d v=e d m$. Clearly, $v$ and $m$ are equivalent measures, and a function $f \in L_{1}(v)$ if and only if $f \cdot e \in L_{1}(m)$. Since $\int T f d v=\int T f \cdot e d m=\int f \cdot e d m=\int f d v$ for every $f \in L_{1}(v)$, it is seen that $T$ is a Markovian operator on $L_{1}(v)$. 
We recall the following definition [9], in which all functions are assumed nonnegative. A function $\tilde{g}$ is a version of $g$ if it is obtained from $g$ as follows: let $g=g_{1}+\bar{g}_{1}, T \bar{g}_{1}=g_{2}+\bar{g}_{2}$, and in general, $T \bar{g}_{i}=g_{i+1}+\bar{g}_{i+1}$, and for some $n$

$$
\tilde{g}=\sum_{i=1}^{n} g_{i}+\bar{g}_{n} .
$$

Remarks. (a) $T^{k-1} g=\sum_{i=1}^{n} T^{k-i} g_{i}+T^{k-n} \bar{g}_{n}$ for $k>n$. Indeed, it follows from $g=g_{1}+\bar{g}_{1}$ and $T \bar{g}_{i}=g_{i+1}+\bar{g}_{i+1}, 1 \leq i \leq n-1$, that for $k>n$ we have

$$
T^{k-1} g=T^{k-1} g_{1}+T^{k-1} \bar{g}_{1},
$$

and

$$
T^{k-i} \bar{g}_{i}=T^{k-i-1} g_{i+1}+T^{k-i-1} \bar{g}_{i+1}, \quad 1 \leq i \leq n-1 .
$$

Adding yields the desired formula.

(b) If $g_{i}\left(\right.$ or $\left.\bar{g}_{n}\right)$ is replaced by a version of $g_{i}$ (or $\left.\bar{g}_{n}\right)$, the result is still a version of $g$. Indeed, let $\tilde{g}=\sum_{i=1}^{n} g_{i}+\bar{g}_{n}$ be a version of $g$; it suffices to consider the case where $g_{1}$ is replaced by a version $\tilde{g}_{1}$, where $\tilde{g}_{1}=\sum_{k=1}^{m} g_{1, k}+\bar{g}_{1, m}, g_{1}=g_{1,1}+\bar{g}_{1,1}$, $T \bar{g}_{1, k}=g_{1, k+1}+\bar{g}_{1, k+1}, 1 \leq k \leq m-1$. If $m \leq n$, let $g_{1}^{\prime}=g_{1,1}, \bar{g}_{1}^{\prime}=\bar{g}_{1}+\bar{g}_{1,1}$; then $T \bar{g}_{1}^{\prime}=T \bar{g}_{1}+T \bar{g}_{1,1}=\left(g_{2}+\bar{g}_{2}\right)+\left(g_{1,2}+\bar{g}_{1,2}\right)$; let $g_{2}^{\prime}=g_{2}+g_{1,2}, \bar{g}_{2}^{\prime}=\bar{g}_{2}$ $+\bar{g}_{1,2}$, and in general, let $g_{i}^{\prime}=g_{i}+g_{1, i}, \bar{g}_{i}^{\prime}=\bar{g}_{i}+\bar{g}_{1, i}$ for $2 \leq i \leq m-1$; $g_{m}^{\prime}=g_{m}+g_{1, m}+\bar{g}_{1, m} ; \bar{g}_{i}^{\prime}=\bar{g}_{i}$ for $i \geq m, g_{i}^{\prime}=g_{i}$ for $i \geq m+1$. Then

$$
\begin{aligned}
\sum_{i=1}^{n} g_{i}^{\prime}+\bar{g}_{n}^{\prime} & =g_{1,1}+\sum_{i=2}^{m-1}\left(g_{i}+g_{1, i}\right)+\left(g_{m}+g_{1, m}+\bar{g}_{1, m}\right)+\sum_{i=m+1}^{n} g_{i}+\bar{g}_{n} \\
& =\left(\sum_{k=1}^{m} g_{1, k}+\bar{g}_{1, m}\right)+\sum_{i=2}^{n} g_{i}+\bar{g}_{n} \\
& =\tilde{g}_{1}+\sum_{i=2}^{n} g_{i}+\bar{g}_{n}
\end{aligned}
$$

is obviously a version of $g$. The case where $m>n$ can be treated similarly.

(c) $\int \tilde{g} d v=\int g d v$. This is clear since $T$ preserves integrals with respect to the measure $v$.

(d) For any integer $N, T^{N} g$ is a version of $g$. To see this, in (2.4) choose $g_{i}=0$ for $1 \leq i \leq N$, then $\bar{g}_{i}=T^{i-1} g, 1 \leq i \leq N$. Let $g_{N+1}=T \bar{g}_{N}=T^{N} g$, and $\bar{g}_{N+1}$ $=0$.

(e) The 'filling scheme': given any two nonnegative functions $w, r \in L_{1}$, let

$$
\begin{array}{ccc}
w_{1}=(w-r)^{+}, & r_{1}=w \wedge r, & \bar{r}_{1}=(w-r)^{-} \\
w_{2}=\left(w_{1}-T \bar{r}_{1}\right)^{+}, & r_{2}=w_{1} \wedge T \bar{r}_{1}, & \bar{r}_{2}=\left(w_{1}-T \bar{r}_{1}\right)^{-} \\
& \cdot & \\
& \cdot & \\
w_{n+1}=\left(w_{n}-T \bar{r}_{n}\right)^{+}, & r_{n+1}=w_{n} \wedge T \bar{r}_{n} & \bar{r}_{n+1}=\left(w_{n}-T \bar{r}_{n}\right)^{-} .
\end{array}
$$


Clearly, $\tilde{r}=r_{1}+\ldots+r_{n}+\bar{r}_{n}$ is a version of $r$. We have for all $i \geq 1, w_{i}+r_{i}$ $=w_{i-1}$, where $w_{0}=w$. Summing over $i, 1 \leq i \leq n$, we obtain $w_{n}+r_{1}+\ldots+r_{n}$ $=w$; hence

$$
\sum_{i=1}^{n} r_{i} \leq w
$$

H. Rost ([12, p. 263]; see also Meyer [6, Theorem 4]) has shown that $\lim _{n} \int \bar{r}_{n} d v=0$ if and only if $\int r \cdot h d v \leq \int w \cdot h d v$ for every deficient $h$; i.e., $h \in L_{\infty}^{+}$and such that $h \leq S h$, where $S$ is the adjoint to $T$ acting on $L_{1}(v)$. Since $T$ is conservative, the only deficient functions are $S$-invariant, hence $\lim _{n} \int \bar{r}_{n} d v$ $=0$ if and only if $\int r \cdot h d v \leq \int w \cdot h d v$ for every $S$-invariant $h \in L_{\infty}^{+}$. In particular, if $0 \leq r \leq T^{N} w$ for some integer $N$ then for each $S$-invariant $h \in L_{\infty}^{+}$,

$$
\int r \cdot h d v \leq \int T^{N} w \cdot h d v=\int w \cdot S^{N} h d v=\int w \cdot h d v
$$

which implies $\lim _{n} \int \bar{r}_{n} d v=0$. Rost [12, p. 265] has also shown that $\lim _{n} \int w_{n} d v$ $=0$ if and only if $\int w \cdot h d v \leq \int r \cdot h d v$ for every excessive, hence in the conservative case $S$-invariant, $h \in L_{\infty}^{+}$.

(f) Let $g \in L_{1}^{+}(m)$. For each $\delta>0$ there is an $\eta>0$ such that if $0 \leq p \leq g$ and $\int p d v<\eta$, then $\int p d m<\delta$.

Proof. Let $\delta>0$. For each $\varepsilon>0$ let $A_{\varepsilon}=\{e>\varepsilon\}$; then $A_{\varepsilon}$ increases to $X$ as $\varepsilon \rightarrow 0$ since $e>0$ on $X$. The Lebesgue dominated convergence theorem implies that there is an $\varepsilon>0$ such that $\int_{A_{\varepsilon}^{c}} g d m<\delta / 2$. Choosing $\eta=\delta \cdot \varepsilon / 2$ we have whenever $0 \leq p \leq g$ and $\int p d v<\eta$ :

$$
\int p d m \leq \int_{A_{\varepsilon}^{c}} g d m+\int_{A_{\varepsilon}} p d m<\frac{\delta}{2}+\frac{1}{\varepsilon} \int_{A_{e}} p e d m \leq \frac{\delta}{2}+\frac{1}{\varepsilon} \int p d v<\delta
$$

We now assume that $f \in L_{1}(m)$ and $\lim _{n} \int\left|T^{n} f\right| d v=0$. Since $\int\left|T^{n} f\right| d v$ $\geq\left|\int T^{n} f d v\right|$ we have $\int f d v=0$. Write $f=g-h, g \geq 0, h \geq 0$, where we may and do assume that $\int g d v=\int h d v=1$. If $\underline{\lim }_{n} \underline{\lim }_{i} \int T^{i}\left|T^{n}(g-h)\right| d m=0$, then for any $\varepsilon>0$ there exist integers $j$ and $N$ such that $\int T^{j}\left|T^{N}(g-h)\right| d m$ $<\varepsilon / M$. It then follows from positivity of $T$ and (2.1) that

$$
\varlimsup_{i} \int\left|T^{i+j+N}(g-h)\right| d m \leq \varlimsup_{i} \int T^{i+j}\left|T^{N}(g-h)\right| d m<\varepsilon
$$

$\varepsilon$ is arbitrary, hence $\lim _{n} \int\left|T^{n}(g-h)\right| d m=0$. We now show that the assumption

$$
\varliminf_{n} \varliminf_{i} \int T^{i}\left|T^{n}(g-h)\right| d m>a>0
$$

leads to a contradiction. 
Let $K$ be an integer larger than $\left(4 M^{3} / a\right) \cdot \int g d m$. Since $g \in L_{1}^{+}(m)$ we can apply remark (f) to obtain an $\eta, 0<\eta<1$, such that if $0 \leq p \leq g$ and $\int p d v<\eta$ then $\int p d m<a / 4 M^{2}$. Since $\lim _{n} \int\left|T^{n}(g-h)\right| d v=0$ by assumption, we can find an integer $N$ such that if $r_{1}=T^{N} g \wedge T^{N} h, T^{N} g=r_{1}+s_{1}$, $T^{N} h=r_{1}+s_{1}^{\prime}$, then

$$
\int r_{1} d v>1-\eta / 4^{K+1} .
$$

Moreover, by choosing $N$ larger if necessary, we obtain from (2.6) that

$$
\varliminf_{i \rightarrow \infty} \int T^{i}\left|T^{N}(g-h)\right| d m=\varliminf_{i \rightarrow \infty} \int T^{i}\left|s_{1}-s_{1}^{\prime}\right| d m>a .
$$

Hence we can assume $\overline{\lim }_{i \rightarrow \infty} \int T^{i} s_{1} d m>a / 2$, and by (2.1) we have

$$
\varliminf_{i \rightarrow \infty} \int T^{i} s_{1} d m>a / 2 M
$$

if (2.8) holds with $s_{1}^{\prime}$ replacing $s_{1}$, the argument is similar.

We next use the 'filling scheme' discussed in remark (e) with $w=g$ and $r=r_{1}$ to find a version $\tilde{r}_{1}=r_{1,1}+\ldots+r_{1, n}+\bar{r}_{1, n}$ of $r_{1}$; it follows from remark (e) that $\lim _{n} \int \bar{r}_{1, n} d v=0$ since $r_{1} \leq T^{N} g$. Let $n$ be such that if $\bar{r}_{1, n}=r_{1}^{\prime \prime}$ then

$$
\int r_{1}^{\prime \prime} d v<\eta / 4^{K+1} \text {. }
$$

Letting $r_{1}^{\prime}=r_{1,1}+\ldots+r_{1, n}$ we have, by remark (c), $\int\left(r_{1}^{\prime}+r_{1}^{\prime \prime}\right) d v=\int r_{1} d v$. It follows from (2.7) and (2.9) that

$$
\int r_{1}^{\prime} d v>1-\eta / 2 \cdot 4^{K}
$$

and, by (2.5),

$$
0 \leq r_{1}^{\prime} \leq g
$$

Write $g=r_{1}^{\prime}+f_{1} ;$ then

$$
\int f_{1} d v=\int g d v-\int r_{1}^{\prime} d v<\eta / 2 \cdot 4^{K}<\eta
$$

and hence $\int f_{1} d m<a / 4 M^{2}$. It follows from (2.1) that

$$
\varlimsup_{i \rightarrow \infty} \int T^{i} f_{1} d m<a / 4 M .
$$

Let us summarize: $\tilde{g}_{1}=T^{N} g=r_{1}+s_{1}$ is a version of $g\left(\right.$ remark (d)); $\tilde{r}_{1}=r_{1}^{\prime}+r_{1}^{\prime \prime}$ is a version of $r_{1}$, hence $r_{1}^{\prime}+r_{1}^{\prime \prime}+s_{1}$ is a version of $g$ by remark (b).

Let $s_{2}=T^{N} r_{1}^{\prime} \wedge s_{1}$. Since $s_{1} \leq T^{N} g=T^{N} r_{1}^{\prime}+T^{N} f_{1}, s_{1} \leq s_{2}+T^{N} f_{1}$. Applying (2.8) and (2.12) we have

$$
\varliminf_{i \rightarrow \infty} \int T^{i} s_{2} d m \geq \varliminf_{i \rightarrow \infty} \int T^{i} s_{1} d m-\varlimsup_{i \rightarrow \infty} \int T^{i} f_{1} d m>a / 4 M .
$$


Write $T^{N} r_{1}^{\prime}=s_{2}+r_{2}$, where $r_{2} \geq 0$ since $s_{2} \leq T^{N} r_{1}^{\prime}$. Moreover, (2.7) implies $\int s_{1} d v<\eta / 4^{K+1}$, hence applying also (2.10) yields

$$
\begin{aligned}
\int r_{2} d v & =\int T^{N} r_{1}^{\prime} d v-\int s_{2} d v \geq \int r_{1}^{\prime} d v-\int s_{1} d v \\
& >1-\eta / 2 \cdot 4^{K}-\eta / 4^{K+1}>1-\eta / 4^{K} .
\end{aligned}
$$

Note that $T^{N} r_{1}^{\prime}=r_{2}+s_{2}$ is a version of $r_{1}^{\prime}$; we have thus obtained a version $\tilde{g}_{2}=r_{2}+r_{1}^{\prime \prime}+s_{1}+s_{2}$ of $g$, where $s_{2}$ satisfies (2.13) and $r_{2}$ satisfies (2.14). Repeating the argument with $r_{2}$ in place of $r_{1}$, we first obtain a version $\tilde{r}_{2}=r_{2}^{\prime}+r_{2}^{\prime \prime}$ of $r_{2}$, with $\int r_{2}^{\prime} d v>1-\eta / 2 \cdot 4^{K-1}, 0 \leq r_{2}^{\prime} \leq g$. Letting $s_{3}$ $=T^{N} r_{2}^{\prime} \wedge s_{1}$ and $T^{N} r_{2}^{\prime}=s_{3}+r_{3}^{*}$ we thus obtain a version $\tilde{g}_{3}$ of $g, \tilde{g}_{3}=r_{3}+r_{1}^{\prime \prime}$ $+r_{2}^{\prime \prime}+s_{1}+s_{2}+s_{3}$, where

$$
\begin{aligned}
\varliminf_{i \rightarrow \infty} \int T^{i} s_{3} d m & >a / 4 M, \\
\int r_{3} d v & >1-\eta / 4^{K-1} .
\end{aligned}
$$

Next obtain a version $\tilde{r}_{3}=r_{3}^{\prime}+r_{3}^{\prime \prime}$ of $r_{3}$ with $\int r_{3}^{\prime} d v>1-\eta / 2 \cdot 4^{K-2}$, $0 \leq r_{3}^{\prime} \leq g$, and let $s_{4}=T^{N} r_{3}^{\prime} \wedge s_{1}, r_{4}=T^{N} r_{3}^{\prime}-s_{4}$, etc.

Continuing the process we obtain a version $\tilde{g}$ of $g$ such that $\tilde{g} \geq s_{1}+s_{2}$ $+\ldots+s_{K}$, where for $1 \leq j \leq K, \varliminf_{i \rightarrow \infty} \int T^{i} s_{j} d m>a / 4 M$. Hence

$$
\varliminf_{i \rightarrow \infty} \int T^{i} \tilde{g} d m>K a / 4 M>M^{2} \cdot \int g d m \text {. }
$$

Let $\tilde{g}=g_{1}+\ldots+g_{n}+\bar{g}_{n}$ be the representation of $\tilde{g}$ as a version of $g$. If $k$ is large, then remark (a) implies

$$
\begin{aligned}
\int T^{k-1} g d m & =\sum_{i=1}^{n} \int T^{k-i} g_{i} d m+\int T^{k-n} \bar{g}_{n} d m \\
& \geq \frac{1}{M}\left[\sum_{i=1}^{n} \int T^{k} g_{i} d m+\int T^{k} \bar{g}_{n} d m\right] \\
& =\frac{1}{M} \int T^{k} \tilde{g} d m>M \cdot \int g d m .
\end{aligned}
$$

This contradicts (2.1) and completes the proof.

It may be worthwhile to point out that Theorem 2.1 also gives some insight into the behavior of iterates of Markovian operators, as may be seen from the following:

Theorem 2.2. Let $T$ be a positive linear contraction operator, conservative and ergodic (hence Markovian) on $L_{1}$ of a $\sigma$-finite measure space $(X, \mathcal{A}, v)$. Let $h$ be a measurable function, positive on $X$ and such that, for each $f$ with $\int|f| \cdot h d v<\infty$,

$$
\sup _{n} \int\left|T^{n} f\right| h d v<\infty .
$$


If $\int|f| h d v<\infty$ and $\lim _{n} \int\left|T^{n} f\right| d v=0$ then $\lim _{n} \int\left|T^{n} f\right| h d v=0$.

Proof. Set $d m=h d v, e=1 / h$. We have $\int T f \cdot e d m=\int f \cdot e d m$ for each $f \in L_{1}(v)$. Since $L_{1}(v) \cap L_{1}(m)$ is dense in $L_{1}(m)$, it follows that $T^{*} e=e$, where $T^{*}$ is the adjoint of $T$ on $L_{1}(m)$. Note that $e$ need not be bounded, but the proof of Theorem 2.1 remains valid; hence $\int\left|T^{n} f\right| d m=\int\left|T^{n} f\right| h d v \rightarrow 0$. Actually, it can be shown that $e$ is bounded, unless $X=Z$, in which case $\lim _{n} \int\left|T^{n} f\right| d m=0$ for each $f$; this follows from the fact that $T^{*}$ admits at most one real-valued invariant function (see e.g. the proof of Theorem 1.2 [11]).

R. V. Chacon [1, Theorem 4] announced the following decomposition theorem for $L_{1}$, which for the sake of simplicity we state here in a slightly less general form (the infinite measure case given in [1] follows by an easy change of measure).

Theorem 2.3 (Chacon). Let $T$ be a positive conservative contraction operator on $L_{1}$ of a probability space $(X, A, v)$. Then $L_{1}$ is the direct sum of Banach spaces $I$ and $\bar{G}$ where $\bar{G}$ is the closure of $G=\left\{g: g=f-T f, f \in L_{1}\right\}$, and $I=\left\{h: h \in L_{1}\right.$, $S h=h\}$, where $S$ is the adjoint of $T$, naturally extended to act on $L_{1}$.

It is known (see e.g. [2]) that $S$-invariant functions are exactly those measurable with respect to the $\sigma$-field $I$ generated by sets $A$ such that $S 1_{A}=1_{A}$. Hence Chacon's theorem may be restated as follows: The class $G$ of functions $g=f-T f, f \in L_{1}$, is dense in the Banach space $L_{0}=\left\{f: f \in L_{1}, E^{J} f=0\right\}$, where $E^{J}$ is the conditional expectation with respect to $\mathscr{J}$. Our improvement consists in showing that $f$ may be required to be in $L_{1}^{+}$rather than in $L_{1}$.

Theorem 2.4. Let $T$ be a positive conservative contraction operator on $L_{1}$ of a $\sigma$ finite measure space $(X, \mathcal{A}, v)$. Then $L_{0}$ is the closure in $L_{1}$ of the class of functions of the form $f-T f, f \in L_{1}^{+}$.

Proof. Let $g \in L_{0}$ and set $g=w-r$, where $w=g^{+}$and $r=g^{-}$. $E^{g} g=0$ implies that

$$
\int w \cdot h d v=\int r \cdot h d v, \quad S h=h \in L_{\infty}^{+} .
$$

Apply the 'filling scheme' described in remark (e) to find a version $\tilde{r}$ of $r$, $\tilde{r}=r_{1}+\ldots+r_{n}+\bar{r}_{n}$. We note that $w=r_{1}+\ldots+r_{n}+w_{n}$. Summing $r_{1}=-\bar{r}_{1}+r$ and $r_{i+1}=-\bar{r}_{i+1}+T \bar{r}_{i}, 1 \leq i \leq n-1$, yields that $\tilde{r}=r-f_{n}+T f_{n}$ where $f_{n}$ $=\bar{r}_{1}+\ldots+\bar{r}_{n-1} \geq 0$. Therefore

$$
\left|g-T f_{n}+f_{n}\right|_{1}=\left|w-r-T f_{n}+f_{n}\right|_{1}=|w-\tilde{r}|_{1}=\left|w_{n}-\bar{r}_{n}\right|_{1}
$$

which converges to zero by remark (e) and (2.18).

3. In this section, we assume that the operator $T$ is induced by a transition measure $T(x, A)$ defined on $X \times \mathcal{A}$; i.e., we assume that 
$T(x, \cdot)$ is a measure on $\mathcal{A}$ for each fixed $x \in X$;

$T(\cdot, A)$ is an $\mathcal{A}$-measurable function for each $A \in \mathcal{A}$.

The transition measures $T^{n}(x, A)$ are defined inductively by:

$$
\begin{aligned}
& T^{0}(x, A)=1_{A}(x), \\
& T^{n}(x, A)=\int T^{n-1}(y, A) T(x, d y),
\end{aligned}
$$$$
x \in X, A \in \mathcal{A}, n=1,2,3, \ldots
$$

We assume that $T$ is null-preserving: if $A \in A, m(A)=0$, then $T(x, A)=0 m$ a.e.; the exceptional null-set where $T(x, A) \neq 0$ may depend on $A$. This assumption guarantees that $T$ defines an operator on $L_{1}(m)$, identified under the Radon-Nikodým map with the space of finite signed $m$-continuous measures on $(X, \mathcal{A})$ :

$$
\nu T(A)=\int T(x, A) \nu(d x), \quad \nu \in L_{1}, A \in \mathcal{A} .
$$

If we let $f=d \nu / d m$, then $T f=d(\nu T) / d m$.

The operator $T^{*}$ adjoint to $T$ on $L_{1}(m)$ acts on $L_{\infty}(m)$ as follows:

$$
T^{*} h(x)=\int T(x, d y) h(y), \quad x \in X, h \in L_{\infty}(m) .
$$

We further assume that the $\sigma$-field $\mathcal{A}$ is separable (=countably generated); it is then possible to find a function $d_{n}(x, y)$, measurable with respect to the product $\sigma$-field $A \times A$, such that

$$
T_{c}^{n}(x, A)=\int_{A} d_{n}(x, y) m(d y)
$$

where, for each $n$ and each $x \in X$,

$$
T^{n}(x, \cdot)=T_{c}^{n}(x, \cdot)+T_{s}^{n}(x, \cdot)
$$

$T_{c}^{n}(x, \cdot)$ is absolutely continuous with respect to $m$, and $T_{s}^{n}(x, \cdot)$ is singular with respect to $m$ (see e.g. [10, p. 226]).

To apply Theorem 2.1, we assume that the operator $T$ induced on $L_{1}(m)$ by $T(x, A)$ satisfies conditions (2.1), (2.3), (3.5) and (3.6):

$$
\begin{aligned}
& \text { For each } n \geq 1 \text { and each } f \in L_{1}^{+}, f \not 0 \text {, } \\
& \text { we have } \sum_{i=0}^{\infty} T^{n i} f(x)=\infty \text { a.e.; }
\end{aligned}
$$

There is a set $B, m(B)>0$, and an integer $n_{0}$, such that for each $x \in B, m\left\{y: d_{n_{0}}(x, y)>0\right\}>0$. 
Condition (3.5) states that every power of $T$ is conservative and ergodic, and implies that $T$ is aperiodic when $T$ is Markovian and (3.6) holds [2]. Condition (3.6) is the 'essential Harris condition' also made in [10], where it is shown that under conditions weaker than those made above there is a measurable function $f_{0}$, unique modulo multiplication by constants, such that $0<f_{0}<\infty$ and $T f_{0}=f_{0}$.

Remark. The following minor corrections are needed in [10]. In Proposition 1 add the assumption that $T$ is conservative and ergodic. On p. 223 the measure $v_{i}$ in (1.1) is defined only on $S_{X_{i}}$. Since every function in $L_{\infty}$ is a countable sum of functions in $S_{X_{i}}, v_{i}$ extends to $v$, invariant on $L_{\infty}$.

We can now state a non-Markovian version of the Jamison-Orey theorem [5]:

Theorem 3.1. Let $T$ satisfy (2.1), (2.3), (3.5) and (3.6). If $f \in L_{1}(m)$ and $\int f \cdot e d m=0$, then $\lim _{n} \int\left|T^{n} f\right| d m=0$. Let $0<f_{0}=T f_{0}<\infty$. If $f_{0} \cdot e$ $\in L_{1}(m)$ then $f_{0} \in L_{1}(m)$ and, for each $f \in L_{1}(m), T^{n} f$ converges in $L_{1}(m)$ to $\left(\int f \cdot e d m / \int f_{0} \cdot e d m\right) \cdot f_{0}$.

Proof. As in $\S 2$, we introduce a new measure $v$ defined by $d v=e \cdot d m$. Then $T$ is a Markovian operator on $L_{1}(v)$. The operator $S$ adjoint to $T$ on $L_{1}(v)$ acts on $L_{\infty}(v)\left(=L_{\infty}(m)\right)$ as follows:

$$
\begin{aligned}
\int f \cdot S h d v & =\int T f \cdot h \cdot e d m=\int f \cdot T^{*}(h \cdot e) d m \\
& =\int f \cdot(1 / e) \cdot T^{*}(h \cdot e) d v
\end{aligned}
$$

hence we have

$$
\operatorname{Sh}(x)=\int \frac{e(y)}{e(x)} T(x, d y) h(y), \quad x \in X, h \in L_{\infty}(v)
$$

and for each $n \geq 1$,

$$
S^{n} h(x)=\int \frac{e(y)}{e(x)} T^{n}(x, d y) h(y)
$$

Set $P(x, A)=S 1_{A}(x)$, then it is easy to see that $P(x, \cdot)$ is a probability measure for each fixed $x \in X ; P(\cdot, A)$ is an $A$-measurable function for each $A \in \mathcal{A}$. Furthermore, if $P^{n}(x, \cdot)=P_{c}^{n}(x, \cdot)+P_{s}^{n}(x, \cdot)$ is the Lebesgue decomposition of $P^{n}(x, \cdot)$ with respect to $v($ or $m)$, then

$$
\begin{aligned}
P_{c}^{n}(x, A) & =\int_{A} \frac{e(y)}{e(x)} d_{n}(x, y) m(d y) \\
& =\int_{A} \frac{1}{e(x)} d_{n}(x, y) v(d y)
\end{aligned}
$$


which shows that $d_{n}^{\prime}(x, y)=d_{n}(x, y) / e(x)$ is the density of $P_{c}^{n}(x, \cdot)$ with respect to the measure $v$. Since $e>0$, for each $x \in X,\left\{y: d_{n_{0}}^{\prime}(x, y)>0\right\}=\left\{y: d_{n_{0}}(x, y)\right.$ $>0\}$; hence with $B$ and $n_{0}$ as in (3.6), we obtain

$$
\text { For each } x \in B, v\left\{y: d_{n_{0}}^{\prime}(x, y)>0\right\}>0 \text {. }
$$

(3.10) shows that the Markovian transition measure $P(x, A)$ satisfies the 'essential Harris condition', and now condition (3.5) implies that the operator $T$ induced by $P(x, A)$ on $L_{1}(v)$ is aperiodic. It follows from the theorem of Jamison and Orey [5], or rather the measure-theoretical form of this theorem [11, Theorem 4.1], that if $f \in L_{1}(v)$ and $\int f d v=0$ then $\lim _{n} \int\left|T^{n} f\right| d v=0$. Apply now Theorem 2.1 to establish that $\lim _{n} \int\left|T^{n} f\right| d m=0$.

Now assume $0<f_{0}=T f_{0}<\infty$ and $f_{0} \cdot e \in L_{1}(m)$; we show that $f_{0}$ $\in L_{1}(m)$. Define a Markovian operator $V$ on $L_{1}(m)$ by $V f=e \cdot T(f / e)$, $f \in L_{1}(m)$. It has been proved in [3, Lemma 1] that $T$ admits a finite equivalent invariant measure if $V$ does. Since $V\left(f_{0} \cdot e\right)=f_{0} \cdot e$, there exists $f_{1} \in L_{1}(m)$ such that $0<f_{1}=T f_{1}$. Hence $f_{0}=$ constant $\times f_{1} \in L_{1}(m)$. Finally, the last assertion of the theorem easily follows from the first one.

Theorem 3.1 may be used to obtain limit theorems for $T^{n}(x, A)$. The existence of a period for $P^{n}(x, A)$ implies the existence of a period for $T^{n}(x, A)$ and, like in the Markovian case there is no essential loss of generality in assuming that the period is 1 , which is implied by (3.5). But it should be noted that the limit theorems for the Markovian kernel $P^{n}(x, A)$, due to $\mathrm{S}$. Orey (positive recurrent case [7]) and N. C. Jain (null recurrent case, see e.g. [7]), do not imply Theorem 3.2 .

The existence of $f_{0}$ appearing below was established in [10] and already used in Theorem 3.1 .

Theorem 3.2. Let $T$ satisfy (2.1), (2.3), (3.5) and (3.6). Let $0<f_{0}=T f_{0}<\infty$.

(a) If $f_{0} \in L_{1}(m)$, then for almost every $x$ the measure $T^{n}(x, \cdot)$ converges in total variation norm to the measure $\left(e(x) / \int f_{0} \cdot e d m\right) f_{0} d m$.

(b) If $f_{0} \notin L_{1}(m)$, then for almost every $x$ and for each set $A$ with $\int_{A} f_{0} d m<\infty$ one has $T^{n}(x, A) \rightarrow 0$.

Proof. (a) Assume at first that for every $x$ there is an integer $N=N(x)$ such that $T_{s}^{N}(x, X)=0$. Then for a fixed $x, T^{N}(x, \cdot)$ is in $L_{1}(m)$ and equals $d_{N}(x, y)=f(y)$. We have $\int f(y) e(y) m(d y)=T^{* N} e(x)=e(x)$, and (a) follows from the last assertion of Theorem 3.1. In general $T_{s}^{n}(x, X)$ of course need not be zero for any $n$, but we will show that it converges to zero for almost every $x$, and this is sufficient to establish (a). Let $R_{n}$ be $T_{s}^{n}(\cdot, \cdot)$ considered as an $L_{\infty}$ operator: $R_{n} h(x)=\int T_{s}^{n}(x, d y) h(y)$. It was kindly shown to us by Dr. Michael Lin that a slight change in Foguel's argument [2, p. 59] yields $R_{n} 1(x)=T_{s}^{n}(x, X)$ $\rightarrow 0$ also in the power-bounded case. Let $g=\lim _{\sup _{n \rightarrow \infty}} R_{n} 1 ;(2.1)$ implies that 
$g \in L_{\infty}$. (Foguel considered the Markovian case, when $R_{n} 1$ decreases, and $g=\lim _{n} R_{n} 1$.) For each integer $k$,

$$
T^{* k} g \geq R_{k} g \geq \varlimsup_{n} R_{k} R_{n} 1 \geq \varlimsup_{n} R_{k+n} 1=g,
$$

by Fatou's lemma and the simple relation $R_{k} R_{n} \geq R_{k+n}$. Hence $T^{* k} g \geq g$ for each $k$, and since $T$ is conservative and ergodic, $T^{* k} g=g=c \cdot e$, where $c$ is a constant (cf. [13]). (3.11) also implies $R_{k} g=g$ for all $k$, hence $\int d_{k}(x, y)$ $g(y) m(d y)=0$ for all $k$ and $x$. Since $e>0$ on $X$ this contradicts (3.6) unless $c=0$. If we let $F=\left\{T^{*} e=e\right\} \cap\left\{R_{n} 1 \rightarrow 0\right\}$, then clearly $m\left(F^{c}\right)=0$ and (a) holds if $x \in F$.

(b) Assume $f_{0} \notin L_{1}(m)$ and let $\pi(E)=\int_{E} f_{0} d m, E \in \mathcal{A}$. We shall show that (b) holds for each $x \in F$. We adapt an argument given by N. C. Jain, who obtained the result (b) in the Markovian case (cf. [7, p. 35]). Assume that (b) is false for some $x_{0} \in F$. Then we can find a set $A$ with $\pi(A)<\infty$, a positive constant $\delta$, and an increasing sequence of integers $\left(n_{k}\right)$ such that for each $k$

$$
T^{n_{k}}\left(x_{0}, A\right) /(\pi(A)+1) \geq \delta .
$$

Using again $T_{s}^{n}(x, X) \rightarrow 0$ for each $x \in F$, we obtain from Theorem 3.1 that for each $x \in F$

$$
\left\|\frac{1}{e\left(x_{0}\right)} T^{n_{k}}\left(x_{0}, \cdot\right)-\frac{1}{e(x)} T^{n_{k}}(x, \cdot)\right\| \rightarrow 0,
$$

and hence

$$
f_{k}(x)=\left|\frac{1}{e\left(x_{0}\right)} T^{n_{k}}\left(x_{0}, A\right)-\frac{1}{e(x)} T^{n_{k}}(x, A)\right| \rightarrow 0 .
$$

We note that $\int e d \pi=\int e f_{0} d m=\infty$ since $\int f_{0} d m=\infty$ (see proof of Theorem 3.1). Using this fact and applying Egorov's theorem we find a set $C$ such that $f_{k}(x) \rightarrow 0$ uniformly on $C$ and $\left(\delta / 2 \cdot e\left(x_{0}\right)\right) \cdot \int_{C} e d \pi>1$. Finally, by choosing $k$ large such that $f_{k}(x)<\left(\delta / 2 \cdot e\left(x_{0}\right)\right)$ on $C$, we have

$$
\begin{aligned}
1 & >\frac{\pi(A)}{\pi(A)+1} \geq \frac{1}{\pi(A)+1} \int_{C} T^{n_{k}}(x, A) \pi(d x) \\
& \geq \frac{1}{\pi(A)+1} \int_{C} e(x)\left(\frac{1}{e\left(x_{0}\right)} T^{n_{k}}\left(x_{0}, A\right)-\frac{\delta}{2 \cdot e\left(x_{0}\right)}\right) \pi(d x) \\
& \geq \frac{\delta}{2 \cdot e\left(x_{0}\right)} \cdot \int_{C} e d \pi
\end{aligned}
$$

which contradicts the choice of $C$.

We have assumed so far (2.3), i.e., that $X=Y$. If $X=Z$ then the situation is much simpler.

Theorem 3.3. Let $T$ be conservative and ergodic, and assume that (3.6) holds and (2.3) fails. Then $\lim _{n} T^{n}(x, X)=0$ for almost every $x$. 
Proof. Since $T$ is conservative and ergodic, that (2.3) fails implies $X=Z$. We note that for each $x$ and $n, T^{n}(x, X)=T^{* n} 1(x)$. If we set $g=\lim _{n \rightarrow \infty}$ sup $T^{* n} 1$ then like in the preceding proof, $T^{*} g=g$ and hence $g=0$ because there exists no $T^{*}$-invariant function different from zero on $Z$. Thus $T^{n}(x, X) \rightarrow 0$ for almost every $x$.

4. We give here a new proof of the Jamison-Orey theorem. As in $\S 3$, let $T$ be an operator on $L_{1}(X, \mathcal{A}, m)$, induced by a null-preserving transition measure $T(x, A)$ and assume that $T(x, X)=1$ for each $x$. It follows that the operator $T$ on $L_{1}(m)$ is Markovian: $\int T f d m=\int f d m$ for $f \in L_{1}(m)$. The decomposition $T^{n}(x, \cdot)=T_{c}^{n}(x, \cdot)+T_{s}^{n}(x, \cdot)$ and the definition of $d_{n}(x, y)$, the density of $T_{c}^{n}(x, \cdot)$, were given in $\S 3$.

Theorem 4.1 (Jamison-Orey). Let $T$ be a Markovian operator on $L_{1}(X, \mathcal{A}, m)$, induced by a transition probability $T(x, A)$ satisfying (3.5) and (3.6). If $f \in L_{1}(m)$ and $\int f d m=0$ then $\lim _{n} \int\left|T^{n} f\right| d m=0$.

Proof. A set $E$ with $m(E)>0$ is called a $C$-set if there exists a positive integer $n$ such that $\inf \left\{d_{n}(x, y) \mid(x, y) \in E \times E\right\}>0$. It is known that under the Harris condition every set of positive measure contains a $C$-set (cf. Orey [7, p. 10]); it follows at once that $X$ is a countable union of $C$-sets. In the aperiodic case, given a $C$-set $E$ there is an integer $N$ such that for $n \geq N$,

$$
\inf _{(x, y) \in E \times E} d_{n}(x, y)=c_{n}>0 .
$$

This discussion of $C$-sets remains valid under the assumptions (3.5), (3.6). (The equivalence of probabilistic and analytic formulations of the Harris condition is known; see Jain [4].) Since subsets of $C$-sets are again $C$-sets, if we show that

$$
\lim _{n} \int\left|T^{n}(g-T g)\right| d m=0
$$

holds for all $g=1_{E}$ where $E$ is a $C$-set with $m(E)<\infty$, it will follow that (4.2) holds for all $g \in L_{1}^{+}$. Therefore, by Theorem $2.4, \lim _{n} \int\left|T^{n} f\right| d m=0$ for all $f$ with $\int f d m=0$.

Set $g=1_{E}$ for some fixed $C$-set $E$, where we may and do assume that $m(E)=1$. It follows from (4.1) that, for $f \in L_{1}^{+}$and $n \geq N$,

$$
T^{n} f(y) \geq \int d_{n}(x, y) f(x) m(d x) \geq c_{n}\left(\int_{E} f d m\right) g(y) .
$$

For a given positive integer $M$ set $q=q(M)=N+M$ and $c=c(M)$ $=(1 / M) \cdot \min \left\{c_{n} \mid N \leq n<q\right\}$, where $c_{n}$ and $N$ are as in (4.1).

Remark. (g) If $f \in L_{1}^{+}$then there is a version $\tilde{f}=\sum_{i=1}^{q} f_{i}+\bar{f}_{q}$ of $f$ with $f_{i}=0$ for $1 \leq i \leq N$ and $f_{i}=c \cdot\left(\int_{E} f d m\right) g$ for $N<i \leq q$. Indeed, we may clearly choose $f_{i}=0$ for $1 \leq i \leq N$ and $\bar{f}_{N}=T^{N-1} f$. By (4.3), 


$$
T \bar{f}_{N}=T^{N} f \geq c_{N} \cdot\left(\int_{E} f d m\right) g \geq M c \cdot\left(\int_{E} f d m\right) g .
$$

Therefore we may let $f_{N+1}=c \cdot\left(\int_{E} f d m\right) g$, and we have

$$
\bar{f}_{N+1}=T \bar{f}_{N}-f_{N+1} \geq\left(\frac{M-1}{M}\right) T \bar{f}_{N}=\left(\frac{M-1}{M}\right) T^{N} f,
$$

which in turn implies by (4.3) that

$$
T \bar{f}_{N+1} \geq \frac{M-1}{M}\left(M c \cdot \int_{E} f d m\right) g=(M-1) c \cdot\left(\int_{E} f d m\right) g .
$$

Therefore we may let $f_{N+2}=c \cdot\left(\int_{E} f d m\right) g$, etc.

We next show that given an $\varepsilon>0$ and a positive integer $M$, there is a version $\tilde{g}=\sum_{i=1}^{n} g_{i}+\bar{g}_{n}$ of $g=1_{E}$, with $\int \bar{g}_{n} d m<\varepsilon$ and such that (A) holds:

(A) For each $i, g_{i}=\sum_{j=1}^{m} g_{i, j}$; for each fixed $j, g_{i, j}=0$ for all $i$ except for a consecutive block of $M$ terms each of which is the same multiple of $g$.

We first apply remark (g) to the function $g$, obtaining a version $\tilde{g}_{1}=\sum_{i=1}^{q} g_{i}$ $+\bar{g}_{q}$ of $g$, where $g_{i}=0$ for $1 \leq i \leq N$, and $g_{i}=c \cdot\left(\int_{E} g d m\right) g=c g$ for $N<i$ $\leq q=N+M$, a consecutive block of $M$ terms each of which is the same multiple of $g$. Hence the $g_{i}$ 's in $\tilde{g}_{1}$ satisfy property (A). The 'filling scheme' described in remark (e) is next applied with $w=g\left(=1_{E}\right)$ and $r=\bar{g}_{q}$, obtaining a version $\hat{g}=\sum_{i=1}^{n} g_{i}^{\prime}+\bar{g}_{n}^{\prime}$ of $\bar{g}_{q}$. Since $0 \leq r=\bar{g}_{q} \leq T^{q-1} g$ and $\int \bar{g}_{q} d m$ $=1-M c$, it follows from remark (e) that there is an integer $n$ such that $\int \bar{g}_{n}^{\prime} d m=(1-M c) \delta$ with $\delta<\frac{1}{2}$. Remark (g) is now applied to each $g_{i}^{\prime}$ to yield a version $\tilde{g}_{i}^{\prime}=\sum_{j=1}^{q} g_{i, j}+\bar{g}_{i, q}$ of $g_{i}^{\prime}$, where $q=N+M, g_{i, j}=0$ for $1 \leq j \leq N$ and $g_{i, j}=c \cdot\left(\int_{E} g_{i}^{\prime} d m\right) g$ which equals $c \cdot\left(\int g_{i}^{\prime} d m\right) g$ for $N<j \leq q$ since $0 \leq g_{i}^{\prime} \leq g=1_{E}$ by the 'filling scheme'. Applying appropriate powers of $T$ to each $\bar{g}_{i, q}$ and $\bar{g}_{n}^{\prime}$, we may combine all the $\bar{g}_{i, q}$ 's and $\bar{g}_{n}^{\prime}$ into some $\bar{g}_{n_{1}}$ for some integer $n_{1}$. By remark (b) $\sum_{i=1}^{q} g_{i}+\sum_{i=1}^{n} \sum_{j=1}^{q} g_{i, j}+\bar{g}_{n_{1}}$ is a version of $g$. Writing this new version in the form $\tilde{g}_{2}=\sum_{i=1}^{n_{1}} g_{i}+\bar{g}_{n_{1}}$, we note that the $g_{i}$ 's satisfy property (A). Moreover,

$$
\begin{gathered}
\int \bar{g}_{n}^{\prime} d m=(1-M c) \delta \\
\sum_{i=1}^{n} \int g_{i}^{\prime} d m=\int \hat{g} d m-\int \bar{g}_{n}^{\prime} d m=\int \bar{g}_{q} d m-\int \bar{g}_{n}^{\prime} d m \\
=(1-M c)-(1-M c) \delta=(1-M c)(1-\delta) ; \\
\int \bar{g}_{i, q} d m=\int \tilde{g}_{i}^{\prime} d m-\sum_{j=1}^{q} \int g_{i, j} d m \\
=\int g_{i}^{\prime} d m-(M c) \cdot \int g_{i}^{\prime} d m=(1-M c) \cdot \int g_{i}^{\prime} d m, \quad 1 \leq i \leq n .
\end{gathered}
$$

(4.4), (4.5), and (4.6) imply 


$$
\begin{aligned}
\int \bar{g}_{n_{1}} d m & =\int\left(\bar{g}_{n}^{\prime}+\sum_{i=1}^{n} \bar{g}_{i, q}\right) d m \\
& =(1-M c) \delta+(1-M c) \cdot \sum_{i=1}^{n} \int g_{i}^{\prime} d m \\
& =(1-M c)(1-M c+M c \delta)<(1-M c)(1-M c / 2)
\end{aligned}
$$

Hence

$$
\int \bar{g}_{n_{1}} d m<(1-M c / 2) \cdot \int \bar{g}_{q} d m
$$

By repeating the process $k$ times, we can obtain a version $\tilde{g}_{k+1}=\sum_{i=1}^{n_{k}} g_{i}+\bar{g}_{n_{k}}$ where the $g_{i}$ 's satisfy property (A). Moreover, the argument used in proving (4.7) also shows that

$$
\int \bar{g}_{n_{k}} d m<(1-M c / 2) \cdot \int \bar{g}_{n_{k-1}} d m
$$

Thus we have shown that given an $\varepsilon>0$ and a positive integer $M$ with $(1 / M)<\varepsilon$, there is a version $\tilde{g}=\sum_{i=1}^{n} g_{i}+\bar{g}_{n}$ of $g$, with $\int \bar{g}_{n} d m<\varepsilon$, and $g_{i}=\sum_{j=1}^{m} g_{i, j}$ where for fixed $j, g_{i, j}=0$ for all $i$ except for a consecutive block of $M$ terms each of which is the same multiple of $g$.

Finally, by remark (a) we have for $k>n$,

$$
\begin{aligned}
T^{k-1} g & =\sum_{i=1}^{n} T^{k-i} g_{i}+T^{k-n} \bar{g}_{n} \\
& =\sum_{j=1}^{m}\left(\sum_{i=1}^{n} T^{k-i} g_{i, j}\right)+T^{k-n} \bar{g}_{n} ; \\
T^{k} g & =\sum_{j=1}^{m}\left(\sum_{i=1}^{n} T^{k-i+1} g_{i, j}\right)+T^{k-n+1} \bar{g}_{n},
\end{aligned}
$$

and hence by property (A) cancellation occurs and

$$
\int\left|T^{k} g-T^{k-1} g\right| d m<(2 / M) \cdot \int g d m+2 \int \bar{g}_{n} d m<4 \varepsilon .
$$

\section{REFERENCES}

1. R. V. Chacon, Resolution of positive operators, Bull. Amer. Math. Soc. 68 (1962), 572-574. MR 25 \#5384.

2. S. R. Foguel, The ergodic theory of Markov processes, Van Nostrand Math. Studies, no. 21, Van Nostrand Reinhold, New York, 1969. MR 41 \#6299.

3. H. Fong, On invariant functions for positive operators, Colloq. Math. 22 (1970), 75-84. MR 42 $\# 8266$.

4. N. C. Jain, A note on invariant measures, Ann. Math. Statist. 37 (1966), 729-732. MR 33 \#4992.

5. B. Jamison and S. Orey, Markov chains recurrent in the sense of Harris, Z. Wahrscheinlichkeitstheorie und Verw. Gebiete 8 (1967), 41-48.

6. P. A. Meyer, Travaux de H. Rost en théorie du potentiel, Université de Strasbourg, Séminaire de Probabilités 1969/70. 
7. S. Orey, Limit theorems for Markov chain transition probabilities, Van Nostrand Math. Studies, no. 34, Van Nostrand Reinhold, New York, 1971.

8.—, An ergodic theorem for Markov chains, Z. Wahrscheinlichkeitstheorie und Verw. Gebiete 1 (1962), 174-176. MR 26 \#3117.

9. D. S. Ornstein, On a theorem of Orey, Proc. Amer. Math. Soc. 22 (1969), 549-551. MR 39 \#6388.

10. D. S. Ornstein and L. Sucheston, On the existence of $a$ o-finite invariant measure under $a$ generalized Harris condition, Contributions to Ergodic Theory and Probability, Lecture Notes in Math., no. 160, Springer-Verlag, Berlin and New York, 1970; pp. 219-233.

11._, An operator theorem on $L_{1}$ convergence to zero, with applications to Markov kernels, Ann. Math. Statist. 41 (1970), 1631-1639. MR 42 \#6938.

12. H. Rost, Markoff-Ketten bei sich füllenden Löchern im Zustandsraum, Ann. Inst. Fourier (Grenoble) 21 (1971), 253-270.

13. L. Sucheston, On the ergodic theorem for positive operators. I, Z. Wahrscheinlichkeitstheorie und Verw. Gebiete 8 (1967), 1-11. MR 35 \#4371.

Department of Mathematics, Bowling Green State University, Bowling Green, Ohio 43402

Department of Mathematics, Ohio State University, Columbus, Ohio 43210 (*)

(*) Current address of both authors. 